\title{
Breve caracterización del Sistema Educativo Mexicano
}

\author{
Brief characterization \\ of the Mexican Educational System
}

Eduardo Backhoff Escudero*

Palabras clave: educación, México, Sistema Educativo Nacional, aprendizaje, resultados educativos

\section{RESUMEN}

En este artículo se describen algunas de las características de mayor importancia del Sistema Educativo Nacional (SEN) en su tramo de educación básica: el contexto social en que se desenvuelve, el tamaño, dimensión y diversidad de las poblaciones que atiende, las inequidades en la oferta educativa, las características de sus docentes y los resultados de aprendizaje. Se destacan los avances que el SEN ha experimentado en los últimos años y los retos a los que se enfrenta para poder cumplir con el mandato constitucional de ofrecer una educación de calidad donde todos los niños y jóvenes del país logren el máximo aprendizaje posible. Se hace énfasis en que la gran deficiencia que tiene la educación de México tiene que ver con los bajos resultados de aprendizaje.

\section{INTRODUCCIÓN}

El propósito de este artículo es describir en números y de una manera breve las características del Sistema Educativo Nacional (SEN) correspondiente al tramo de la educación obligatoria. Lo anterior con el fin de apreciar su dimensión y diversidad, así como algunos de los grandes problemas que enfrenta para poder cumplir con el espíritu del artículo tercero de la Constitución Política de los Estados Unidos Mexicanos (CPEUM), sobre la obligación del Estado de garantizar el derecho de niños y jóvenes a recibir una educación de calidad con equidad.

\footnotetext{
*Métrica Educativa, A.C.; ebackhoff@gmail.com
}

Key words: education, Mexico, National Educational System, learning and educational outcomes \begin{abstract}
features of the Mexican Educational System (MES)

in its basic level: the social context in which it population it serves; the inequities in the educational supply, characteristics of its teachers and that the MES has experienced in recent years and the challenges faced in order to comply with the constitutional mandate to provide educational the country will achieve the maximum possible learning. It emphasizes on the fact that the big deficiency of Mexican education is related to the low learning outcomes.
\end{abstract}

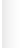


Para lograr este propósito, el texto aborda los siguientes seis apartados. En el primero se describe el contexto socioeconómico del país en el cual opera el SEN, poniendo énfasis en su crecimiento poblacional, la desigualdad de ingreso económico, los niveles de pobreza, el porcentaje de población indígena y el tamaño de las poblaciones donde habitan los mexicanos a lo largo y ancho del país. En el segundo se presenta el crecimiento poblacional de niños y jóvenes que tienen la edad de asistir a los diversos niveles educativos de la educación obligatoria, de acuerdo con su lugar de residencia (urbano o rural). En el tercer apartado se describe la composición del SEN en cuanto al tipo y número de escuelas, de estudiantes y de maestros que lo conforman, así como el nivel educativo de la población mexicana de 15 años o más. En el cuarto se describen algunas de las características de la oferta educativa de las escuelas, poniendo énfasis en algunas de las carencias básicas. En el quinto se caracteriza al docente mexicano, en cuanto a sus estudios, edad y sexo. En el sexto y último apartado se analizan algunos de los resultados educativos de los estudiantes, tanto en las evaluaciones de aprendizaje nacionales como en las internacionales. En este apartado también se analizan los factores socioeconómicos y culturales que se asocian al logro educativo de los alumnos. El artículo termina con una sección de conclusiones, donde se hace un balance de la problemática del SEN.

Es importante comentar que este trabajo se fundamenta, principalmente, en cuatro informes, publicados por el Instituto Nacional de Geografía y Estadística (INEGI) y el Instituto Nacional para la Evaluación de la Educación (INEE): Principales resultados de la encuesta intercensal (INEGI, 2015), La educación obligatoria en México (INEE, 2018), Panorama Educativo de México (INEE, 2017) y Segundo Estudio Internacional sobre Enseñanza y Aprendizaje (TALIS, 2013): resultados de México (Backhoff y Pérez-Morán, 2015).

\section{EL CONTEXTO NACIONAL DEL SISTEMA EDUCATIVO}

De acuerdo con la última Encuesta Intercensal, realizada por el Instituto Nacional de Geografía y Estadística (INEGI, 2015), se estima que en 2015 residían en México 119 millones 530 mil 
753 personas, de las cuales $51.43 \%$ eran mujeres. Asimismo, se calcula que la tasa de crecimiento poblacional promedio del país de 2010 a 2020 será de 1.4 . Igualmente, mientras que la mediana en la edad de la población mexicana en 2000 era de 22 ańos, en 2015 fue de 27, lo que indica un envejecimiento de la población, que, sin embargo, sigue siendo predominantemente joven. Mientras que la población de 0 a 14 ańos ha disminuido, la de 65 o más ha crecido.

Por otro lado, hay que tomar en cuenta que el país se desenvuelve en un contexto de pobreza económica e inequidad social, con sus grandes diferencias entre entidades federativas. El cuadro1 presenta el porcentaje de la población mexicana que vivía en distintas condiciones de pobreza en 2008 y 2016. Aquí se puede observar que, aunque el país ha avanzado en este indicador, sigue habiendo una cantidad importante de personas que padecen de una pobreza extrema o moderada (43.6\%); sólo el $22.6 \%$ de la población no es pobre ni vulnerable (INEE, 2018).

CUADRO 1. Porcentaje de la población en condiciones de pobreza

\begin{tabular}{|l|c|c|}
\hline \multicolumn{1}{|c|}{ Condiciones de pobreza } & 2008 & 2016 \\
\hline Pobreza extrema & 10.6 & 7.7 \\
\hline Pobreza moderada & 33.7 & 35.9 \\
\hline Vulnerable por carencias sociales & 32.2 & 26.8 \\
\hline Vulnerable por ingresos & 4.7 & 7.0 \\
\hline No pobre y no vulnerable & 18.8 & 22.6 \\
\hline Total & 100.0 & 100.0 \\
\hline
\end{tabular}

Fuente: INEE. Informe 2018: La educación obligatoria en México. México: INEE.

Además de la pobreza, el país padece una gran desigualdad en la distribución de los ingresos de su población económicamente activa. El cuadro 2 muestra las diferencias en ingreso de las personas, de acuerdo con el quintil en que se ubicaban. Así, en 2016, la quinta parte de la población más pobre (quintil I), ganaba en promedio $\$ 825.00$ pesos al mes, mientras que la del quintil $\mathrm{V}$ gana $\$ 10542.00$ En este cuadro también se podrá apreciar que 
los salarios entre 2008 y 2016 no han variado significativamente estas diferencias (INEE, 2018).

\section{CUADRO 2. Ingreso promedio per cápita por quintil de ingreso}

\begin{tabular}{|l|r|r|}
\hline \multicolumn{1}{|c|}{ Quintil } & 2008 & 2016 \\
\hline I & 696 & 825 \\
\hline II & 1546 & 1633 \\
\hline III & 2424 & 2428 \\
\hline IV & 3794 & 3691 \\
\hline V & 10416 & 10542 \\
\hline Total & 3775 & 3824 \\
\hline
\end{tabular}

Fuente: INEE. Informe 2018: La educación obligatoria en México. México: INEE.

Por otro lado, el país se caracteriza por tener una población que vive de manera concentrada en grandes ciudades $y$, a la vez, en una gran cantidad de poblaciones rurales y pequeñas. El cuadro 3 muestra que hasta 2010, el país contaba con poco más de 192 mil localidades, de las cuales 139 mil tenían menos de 100 habitantes y 36 más de medio millón (INEE, 2018).

CUADRO 3. Número de localidades por tamaño de población (2010)

\begin{tabular}{|l|c|}
\hline \multicolumn{1}{|c|}{ Tamaño de localidad } & Número de localidades \\
\hline Menos de 100 hab. & 139158 \\
\hline 100 a 499 hab. & 34253 \\
\hline 500 a 999 hab. & 9264 \\
\hline 1000 a 2499 hab. & 5921 \\
\hline 2500 a 14999 hab. & 3021 \\
\hline 15000 a 99999 hab. & 499 \\
\hline 100000 a 499999 hab. & 95 \\
\hline Más de 500000 hab. & 36 \\
\hline Total & 192247 \\
\hline
\end{tabular}

Fuente: INEE. Informe 2018: La educación obligatoria en México. México: INEE. 
Finalmente, otra característica importante del país es la cantidad de su población indígena. Como se muestra en la figura 1 , es de aproximadamente 12 millones, cantidad que representa $10.1 \%$ de los mexicanos. De la población mayor de tres años (93.5\% de la población total), 7 millones 382 mil 785 (6.5\%) son hablantes de una lengua indígena y, de éstos, 909 mil 356 (12.3\%) son monolingues (INEGI, 2015).

FIGURA 1. Población indígena en los hogares (2015)

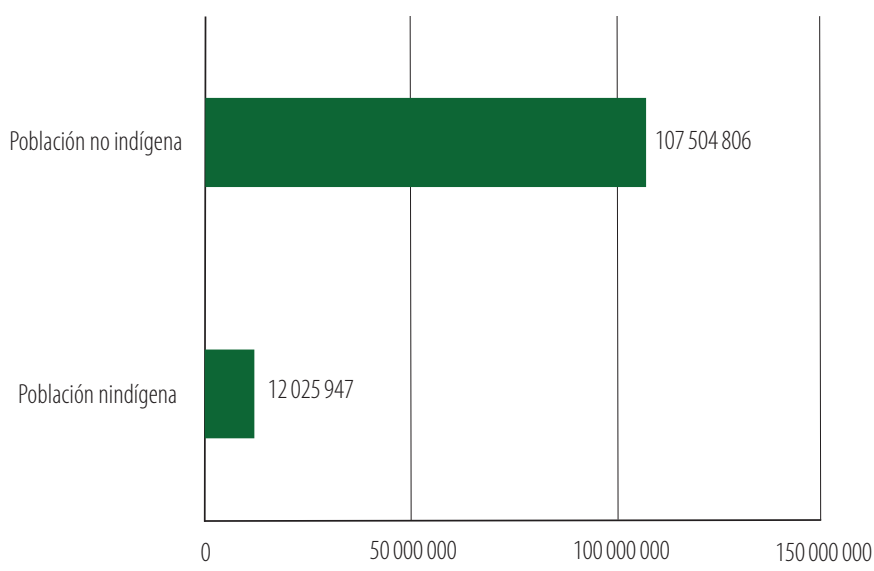

Fuente: INEGI. Principales resultados de la Encuesta Intercensal 2015. Estados Unidos Mexicanos. México: INEGI.

\section{POBLACIONES EN EDAD DE ASISTIR A LA ESCUELA}

Entre 2000 y 2015 el número de niños y jóvenes menores de 18 ańos aumentó, pero su peso entre el total de la población disminuyó; en el mismo periodo de tiempo, los nińos de 3 a 11 años no sólo redujeron su proporción, sino que hubo una baja en términos absolutos de la población. Lo anterior da cuenta de un cambio en la estructura etaria de los mexicanos en la que la población de nińos y jóvenes ha ido descendiendo paulatinamente.

En lo que respecta a la población entre 15 y 17 años -edad idónea para cursar la Educación Media Superior (EMS)-, su peso relativo también ha disminuido: de $6.3 \%$ en 2000 a $5.4 \%$ en 2015. Sin embargo, en términos absolutos ha presentado un cre- 
cimiento promedio anual de $0.4 \%$ en el periodo 2000-2015 (por debajo del crecimiento total de la población; INEE, 2018).

CUADRO 4. Población y porcentaje de habitantes por grupos de edad

\begin{tabular}{|l|c|c|c|c|}
\hline \multirow{2}{*}{ Grupo de edad } & \multicolumn{2}{|c|}{2000} & \multicolumn{2}{c|}{2015} \\
\cline { 2 - 5 } & Cantidad & Porcentaje & Cantidad & Porcentaje \\
\hline 3 a 5 años & 6696125 & 6.9 & 6563407 & 5.5 \\
\hline 6a 11 años & 13308515 & 13.7 & 13224826 & 11.1 \\
\hline 12 a 14 años & 6392415 & 6.6 & 6813683 & 5.7 \\
\hline 15 a 17 años & 6123804 & 6.3 & 6462851 & 5.4 \\
\hline 18 a 24 años & 12939465 & 13.3 & 14975262 & 12.5 \\
\hline
\end{tabular}

Fuente: INEE. Informe 2018: La educación obligatoria en México. México: INEE.

Por otro lado, de acuerdo con el INEE (2018) la escolaridad de la población del país ha ido en aumento en las últimas décadas (figura 2). Mientras que en 1960 la población de 15 años y más alcanzó una escolaridad promedio de apenas 2.6 grados escolares, en 2015 ésta se incrementó a 9.2 grados (equivalente a la secundaria terminada), lo que en promedio equivale a un aumento de 1.1 grados por década. Sin embargo, la escolaridad no es igual en todas las poblaciones. Por ejemplo, la más pobre, que se ubica en el primer quintil de capital económico, apenas alcanza una escolaridad promedio de 6.6 grados, mientras que la del quinto quintil (población más rica) logra una escolaridad de 12.2 grados; es decir, prácticamente el doble.

En cuanto a la población analfabeta, el país ha avanzado considerablemente. Así, mientras que en 1990, 12.4\% de la población mayor de 15 años era analfabeta, en 2015 esta cifra bajó a $5.5 \%$. Este decremento se traduce, en promedio, a 3 puntos porcentuales por década (figura 3). Sin embargo, los distintos grupos poblacionales y entidades del país también difieren enormemente en este indicador educativo (INEE, 2018). 
FIGURA 2. Grado promedio de escolaridad de la población mexicana de 15 años o más

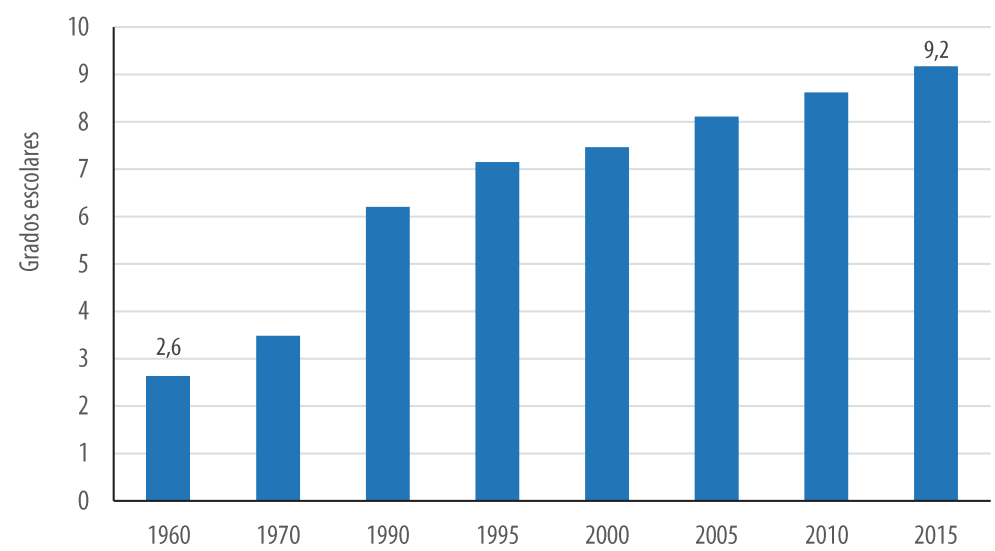

Fuente: INEE. Informe 2018: La educación obligatoria en México. México: INEE.

FIGURA 3. Porcentaje de población mexicana analfabeta de 15 años o más

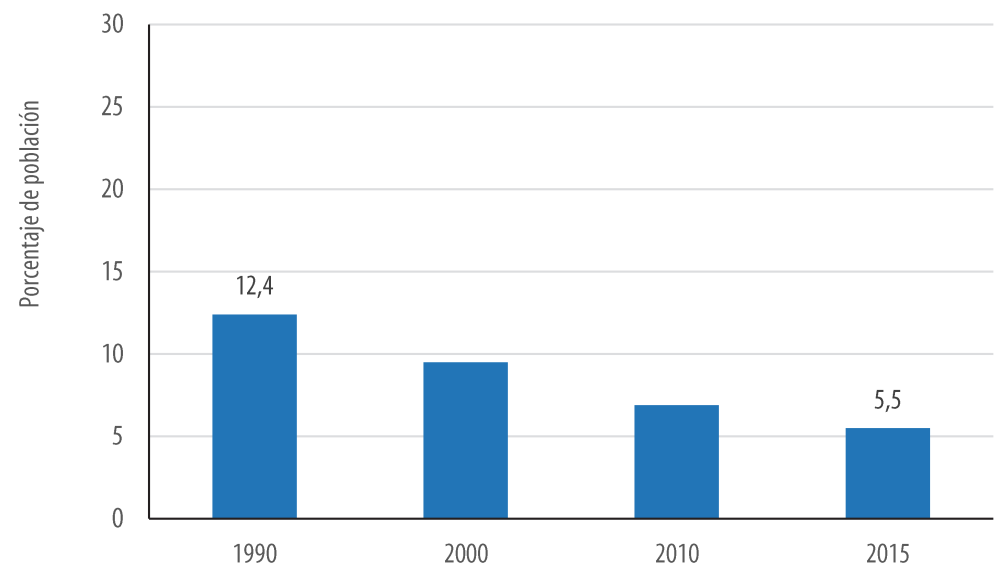

Fuente: INEE. Informe 2018: La educación obligatoria en México. México: INEE.

\section{COMPOSICIÓN DE LA EDUCACIÓN OBLIGATORIA}

El SEN se caracteriza por su gran tamaño y por la diversidad de su oferta educativa. En el cuadro 5 se muestra una clasificación 
de los tipos de servicios educativos que se ofrecen en la educación obligatoria, de acuerdo con el nivel escolar de que se trate (preescolar, primaria, secundaria y EMS). En este cuadro se puede apreciar el número de alumnos, docentes y centros escolares que conforman la educación obligatoria del país. En total, en el ciclo escolar 2015-2016, la educación obligatoria estaba conformada por cerca de 30.8 millones de alumnos, 1.5 millones de docentes y 243.6 mil escuelas. Por el número de alumnos y escuelas, el nivel educativo más grande es el de primaria; mientras que el más pequeño, por su número de alumnos, es el preescolar, seguido por la EMS (INEE, 2017).

CUADR0 5. Número de alumnos, docentes y escuelas por nivel y tipo educativo: ciclos escolares 2015-2016

\begin{tabular}{|c|c|c|c|c|}
\hline Nivel educativo & Tipo de servicio & Alumnos & Docentes & Escuelas \\
\hline \multirow[t]{4}{*}{ Preescolar } & General & 4237107 & 194058 & 60951 \\
\hline & Indígena & 412830 & 18818 & 9804 \\
\hline & Comunitario & 162029 & 17905 & 18654 \\
\hline & Total & 4811966 & 230781 & 89409 \\
\hline \multirow[t]{4}{*}{ Primaria } & General & 13321710 & 525120 & 77313 \\
\hline & Indígena & 818349 & 36952 & 10180 \\
\hline & Comunitario & 110366 & 12138 & 10511 \\
\hline & Total & 14250425 & 574210 & 98004 \\
\hline \multirow[t]{6}{*}{ Secundaria } & General & 3448931 & 227630 & 12052 \\
\hline & Técnica & 1869327 & 101019 & 4711 \\
\hline & Telesecundaria & 1448281 & 72954 & 18667 \\
\hline & Para trabajadores & 25209 & 2820 & 243 \\
\hline & Comunitaria & 43497 & 4154 & 3212 \\
\hline & Total & 6835245 & 408577 & 38885 \\
\hline \multirow[t]{4}{*}{ EMS } & Bachillerato general & 3096442 & 199128 & 14202 \\
\hline & Bachillerato tecnológico & 1820178 & 92183 & 2799 \\
\hline & Profesional técnico & 68460 & 8608 & 632 \\
\hline & Total & 4985080 & 292484 & 17400 \\
\hline \multicolumn{2}{|l|}{ Total } & 30882716 & 1506052 & 243698 \\
\hline
\end{tabular}

Fuente: INEE (2017). Panorama Educativo de México. México: INEE. 
Por otro lado, la asistencia escolar de la población mexicana en edad de cursar cada nivel educativo se ha ido incrementando a lo largo de los años, lo que se puede observar con toda claridad en la figura 4, que muestra el porcentaje de escolares que han asistido a la escuela de 1990 a 2015. Igualmente, es importante hacer notar que, en el caso de la primaria, la asistencia de los niños de 6 a 11 años es muy cercana al 100\%. Sin embargo, todavía una quinta parte de los nińos no asiste a preescolar, mientras que la cuarta parte de los jóvenes mexicanos no asiste a EMS (INEE, 2018).

FIGURA 4. Tasa de asistencia escolar según grupos de edad

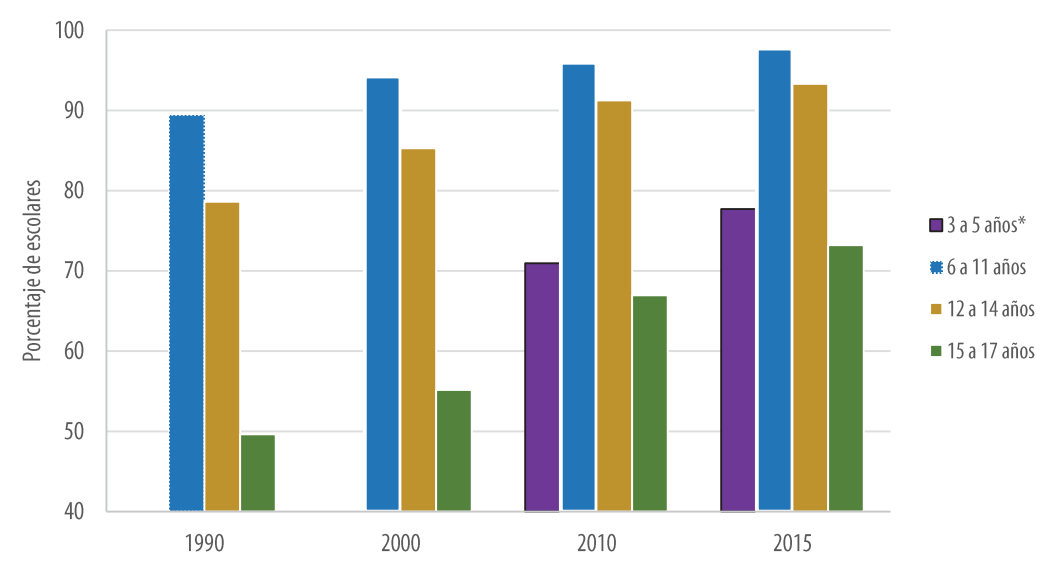

Fuente: INEE. Informe 2018: La educación obligatoria en México. México: INEE.

\section{OFERTA EDUCATIVA}

Los recursos escolares en México todavía son escasos y no se distribuyen bajo un principio de equidad (INEE 2017). Una proporción considerable de centros educativos no cuenta con los espacios necesarios para ofrecer a los estudiantes las mínimas oportunidades de aprendizaje y de bienestar escolar. Por ejemplo, como se muestra en el cuadro 6 , muchas escuelas carecen de biblioteca, sala de cómputo, espacio deportivo, área recreativa, 
laboratorios y sanitarios. Más aún, una de cada 100 escuelas no cuenta con aulas para impartir clases. Asimismo, alrededor de uno de cada 10 preescolares y primarias no dispone de un sanitario en su inmueble, a pesar de que es una condición básica de higiene para la comunidad escolar.

Si bien es cierto que la inmensa mayoría de escuelas públicas cuenta con aulas para impartir clases, también lo es que entre 1 y $2 \%$ no cuenta con este espacio esencial de cualquier centro educativo. Esto sin contar que, de acuerdo con el INEE (2017), en cerca de $40 \%$ de las escuelas se observan problemas de goteras o filtraciones de agua, o bien fisuras o cuarteaduras graves en techos, muros o pisos.

De acuerdo con el INEE (2017), las escuelas a las que asisten alumnos de contextos socioeconómicos menos favorecidos -como las indígenas, las comunitarias, las telesecundarias y los telebachilleratos- cuentan con una menor infraestructura y ésta es de menor calidad.

CUADRO 6. Porcentaje de escuelas públicas con carencias de espacios educativos

\begin{tabular}{|l|c|c|c|c|c|c|}
\hline Nivel educativo & Aulas & Biblioteca & Sala de cómputo & $\begin{array}{c}\text { E. deportivos } \\
\text { orecreativos }\end{array}$ & Laboratorios & Sanitarios \\
\hline Preescolar* & 1.2 & 24.1 & 83.6 & 24.8 & NA & 11.5 \\
\hline Primaria* & 1.0 & 32.0 & 68.9 & 34.0 & NA & 9.4 \\
\hline Secundaria* & 1.1 & 27.6 & 57.2 & 31.2 & 24.0 & 6.4 \\
\hline EMS** $^{*}$ & 2.5 & 53.0 & 39.4 & 27.3 & 59.5 & 3.1 \\
\hline
\end{tabular}

* Información de 2013. ** Información de 2016; NA: no aplica.

Fuente: INEE (2017). Panorama Educativo de México. México: INEE.

Por otro lado, una herramienta fundamental para el proceso de enseńanza aprendizaje que todo centro educativo debe poseer y poner a disposición de maestros y estudiantes es el equipo de cómputo con acceso a Internet. Al igual que en el caso de los espacios físicos, una gran cantidad de centros escolares mexicanos carece de esta herramienta, situación que se muestra en el cuadro 7 para los distintos niveles educativos y centros escolares por tipo de sostenimiento. Queda claro que las escuelas de sostenimiento privado están mejor equipadas que las de sostenimiento público. 
CUADRO 7. Porcentaje de escuelas con al menos una computadora para uso educativo y con Internet

\begin{tabular}{|l|c|c|c|}
\hline \multirow{2}{*}{ Nivel educativo } & Sostenimiento & Almenos una computadora & Escuelas con Internet \\
\hline \multirow{2}{*}{ Primaria* } & Público & 35.3 & 48.0 \\
\hline \multirow{2}{*}{ Secundaria* } & Privado & 85.0 & 96.4 \\
\cline { 2 - 4 } & Público & 64.9 & 55.9 \\
\hline \multirow{2}{*}{ EMS** $^{*}$} & Privado & 92.6 & -- \\
\cline { 2 - 4 } & Público & 75.6 & 70.3 \\
\cline { 2 - 4 } & Privado & 86.0 & 95.6 \\
\hline
\end{tabular}

* Información de 2013. ** Información de 2016, -- sin información Fuente: INEE (2017). Panorama Educativo de México. México: INEE.

\section{DOCENTES}

Los docentes representan el componente más importante del SEN. De ellos depende, en gran medida, que los estudiantes aprendan los contenidos de los planes y programas de estudio y que adquieran aprendizajes significativos. Por supuesto, se requiere contar además con muchos otros elementos para lograr metas ambiciosas de aprendizaje, entre ellos: una organización escolar eficiente; un clima escolar adecuado; un ambiente propicio para el aprendizaje, así como infraestructura, equipamiento y materiales educativos funcionales y adecuados. Si bien todos estos elementos son importantes, también lo es que, aun contando con ellos, es imposible que los estudiantes logren resultados de aprendizaje satisfactorios sin profesores bien formados.

A pesar de la importancia de los docentes, hay muy pocos estudios nacionales e internacionales que los puedan caracterizar. Si bien se han realizado estudios de corte cualitativo para conocer una diversidad de aspectos de los docentes, el primero internacional que se diseñó con este propósito fue el Estudio Internacional de Enseñanza y Aprendizaje (TALIS, por sus siglas en inglés), coordinado por la OCDE. Este estudio tipo encuesta se enfocó, en 2008, a conocer las diversas características de los docentes de segundo de secundaria. Sin embargo, en 2013 amplió la posibilidad de que los países también pudieran estudiar a los docentes de primaria y de EMS, opción por la que optó México. 
A continuación, se hace un recuento de algunas de las características sociodemográficas, educativas y laborales de los docentes mexicanos de estos tres niveles educativos, con base en el segundo estudio de TALIS (Backhoff y Pérez-Morán, 2015).

- Sexo. Más de la mitad de los docentes son mujeres, lo que es especialmente cierto para el caso de primaria, donde cerca de 7 de cada 10 docentes son del sexo femenino. En los casos de la secundaria y de la EMS la proporción se acerca a 50\%.

- Edad. El promedio de edad de los docentes mexicanos es cercano a los 41 años. Sin embargo, son más jóvenes los docentes de primaria, seguidos de los de EMS y, finalmente, los de secundaria. Cerca de $50 \%$ de quienes laboran en primaria tienen menos de 40 años, en esta condición se encuentra el $45 \%$ de los docentes de EMS y $42 \%$ de maestros de secundaria.

- Escolaridad y preparación. Alrededor de $20 \%$ de los docentes de primaria no tiene el nivel de licenciatura, $10 \%$ en secundaria y $8 \%$ en EMS. Asimismo, el porcentaje de profesores que completaron un programa de formación docente que trabajan en la primaria es ligeramente mayor a $80 \%$, en secundaria a $60 \%$ y en EMS a $25 \%$. En cuanto a la preparación para ejercer su función docente, en opinión de los profesores, quienes trabajan en primaria se sienten mejor preparados que los de secundaria, y éstos que los de EMS. Entre 70 y $80 \%$ de los profesores reporta estar "bien" o "muy bien" preparados en contenidos, didáctica y práctica de enseñanza. Sin embargo, una cantidad importante de profesores $(20 \%$ en primaria, $60 \%$ en secundaria y $40 \%$ en EMS) reporta que no imparte la o las asignaturas en las que ellos fueron formados.

- Empleo y experiencia laboral. El porcentaje de docentes que está empleado de tiempo completo es cercano a $65 \%$ en primaria, $45 \%$ en secundaria y $35 \%$ en EMS. El resto trabaja de tiempo parcial. En primaria, 5\% tiene medio turno o menor número de horas, en secundaria trabaja en esta condición $20 \%$ los maestros, mientras que en EMS la cifra es cercana a $35 \%$. Por otro lado, en primaria, más de $70 \%$ de docentes tiene un empleo permanente; en secundaria, casi 8 de cada 10 y en EMS $60 \%$. Finalmente, en números redondos, el prome- 
dio de años de experiencia laboral de los docentes de primaria y de secundaria es cercana a los 16 años, mientras que en EMS es de 14 ańos. Sin embargo, los años de laborar en la escuela donde actualmente trabajan los docentes es de ocho años en primaria, de diez años en secundaria y de 11 en EMS.

- Tamaño de las escuelas y de las clases. En promedio, las escuelas primarias de México tienen 195 estudiantes, las de secundaria 246 y las de EMS 380. Asimismo, el tamaño promedio de una clase en primaria es de 26 alumnos, en secundaria de 30 y en EMS de 34 estudiantes.

- Calidad de los docentes y cumplimiento de la normatividad. De acuerdo con la opinión de los directores, la escasez de docentes bien preparados o con un buen desempeño pedagógico en primaria es cercana a $32 \%$, en secundaria a $50 \%$ y en EMS a $36 \%$. Finalmente, dos problemas escolares que se consideran importantes se relacionan con los retardos de los docentes a clases y su ausentismo del centro escolar. En opinión de los directores, estos problemas son mayores en la secundaria que en la primaria. En secundaria, al menos una vez por semana, $22 \%$ de profesores llega tarde y poco más de $15 \%$ se ausenta de su centro escolar. En primaria el problema es menor, ya que estas cifras son $5 \%$ y $2 \%$, respectivamente.

\section{RESULTADOS EDUCATIVOS Y CONTEXTO SOCIAL}

El sentido de la educación es lograr que los futuros ciudadanos adquieran los conocimientos, habilidades y competencias que les permitan realizarse como personas y que sean capaces de contribuir a que el país se desarrolle social y económicamente, de acuerdo con su cultura, contexto y valores democráticos. Por ello, un indicador fundamental de la calidad de un sistema educativo es el grado en que sus estudiantes adquieren los aprendizajes esperados en cada uno de los niveles educativos, desde el preescolar hasta la educación superior. Para estimar el grado en que se está cumpliendo con este indicador, los países participan en diversos estudios nacionales e internacionales que evalúan el nivel de rendimiento académico de sus estudiantes. 
En el caso de México, el INEE desarrolla las evaluaciones nacionales; antes, se hacía con una batería de pruebas conocidas como Excale (Exámenes para la Calidad y el Logro Educativos) y, actualmente, con pruebas de conocimientos similares conocidas como Planea (Plan Nacional para la Evaluación de los Aprendizajes). Las actuales evaluaciones de logro académico que realiza el INEE están diseñadas para conocer el grado en que los estudiantes dominan los aprendizajes básicos (por ahora, Lenguaje y comunicación y Matemáticas) que se establecen en los planes y programas de estudio nacionales, de los grados terminales de $3^{\circ}$ de preescolar, $6^{\circ}$ de primaria, $3^{\circ}$ de secundaria y último grado de EMS. México también participa en los estudios internacionales de PISA ${ }^{1}$ (estudiantes de 15 años), LLECE $^{2}$ (estudiantes de $3^{\circ} \mathrm{y}$ $6^{\circ}$ de primaria) e $\operatorname{ICCS}^{3}$ (estudiantes de $2^{\circ}$ de secundaria). En su conjunto, todas estas evaluaciones de aprendizaje sirven para evaluar la eficacia del SEN, en cuanto a los aprendizajes que logran los estudiantes en distintas asignaturas y grados escolares.

Dado que sería muy extenso describir los resultados de todas estas evaluaciones, a continuación sólo se resumirán, a manera de ejemplo, los que representan cada nivel educativo. Con ellos se podrá estimar el grado en que el SEN está cumpliendo con sus metas de aprendizaje. El cuadro 8 muestra el porcentaje de estudiantes que se ubica en cada uno de los cuatro niveles de logro educativo que utiliza el INEE para reportar sus resultados, donde: el primero, NI, indica que el estudiante presenta un nivel insuficiente de aprendizaje; el segundo nivel, NII, indica que el aprendizaje del estudiante es apenas básico; en el tercer nivel, NIII, los estudiantes dominan suficientemente bien los contenidos curriculares y, finalmente, en el NIV se ubican los estudiantes que dominan con maestría las competencias escolares evaluadas.

\footnotetext{
${ }^{1}$ PISA (Programa de Evaluación de Estudiantes Internacionales), a cargo de la OCDE, evalúa básicamente, tres dominios escolares: comprensión de lectura, matemáticas y ciencias naturales.

${ }^{2}$ LLECE (Laboratorio Latinoamericano de Evaluación de la Calidad de la Educación), a cargo de la oficina de la OREALC/UNESCO, evalúa comprensión de lectura y matemáticas)

${ }^{3}$ ICSS (Estudio Internacional de Educación Cívica y Ciudadana), a cargo de la IEA (Asociación Internacional de Evaluación del Aprendizaje).
} 
CUADRO 8. Porcentaje de estudiantes que se ubica en cada uno de los niveles de logro de Planea por nivel escolar y asignatura

\begin{tabular}{|l|r|r|r|r|r|r|r|r|r|}
\hline \multirow{2}{*}{ Nivel escolar } & \multirow{2}{*}{ Año } & \multicolumn{4}{|c|}{ Lenguaje y comunicación } & \multicolumn{4}{|c|}{ Matemáticas } \\
\cline { 3 - 10 } & & NI & NII & NIII & NIV & NI & NII & NIII & NIV \\
\hline 30 Preescolar & 2011 & 6 & 42 & 34 & 17 & 9 & 50 & 27 & 14 \\
\hline 60 Primaria & 2015 & 50 & 33 & 15 & 3 & 61 & 19 & 14 & 7 \\
\hline 30 Secundaria & 2017 & 34 & 40 & 18 & 8 & 65 & 22 & 9 & 5 \\
\hline Último EMS & 2017 & 34 & 28 & 29 & 9 & 66 & 23 & 8 & 3 \\
\hline
\end{tabular}

Fuente: INEE (2017). Panorama Educativo de México. México: INEE.

En este cuadro podremos apreciar, como lo señala el INEE (2018), que:

- Los estudiantes mexicanos tienen un mejor nivel de dominio en Lenguaje y comunicación que en Matemáticas.

- Los niveles de dominio son más altos en preescolar que en el resto de los niveles educativos.

- A partir de $6^{\circ}$ de primaria, se observan deficiencias severas en los aprendizajes, considerando el alto porcentaje de estudiantes que se ubican en el nivel insuficiente (NI) y el bajo porcentaje que se ubica en el nivel más alto de logro educativo (NIV).

- Al término de la educación secundaria y de la EMS, dos de cada tres estudiantes no dominan las habilidades de Matemáticas y en la misma situación se encuentra uno de cada tres alumnos en Lenguaje y comunicación.

Por otro lado, los resultados de aprendizaje están muy ligados a las condiciones socioeconómicas y culturales de los estudiantes. Así, los resultados de aprendizaje más altos se observan en estudiantes pertenecientes a clases sociales privilegiadas; lo contrario sucede con los alumnos que provienen de contextos deprimidos social y económicamente. Esto se muestra en la figura 5, en la que se puede observar una relación muy estrecha entre las puntuaciones de Planea de Lenguaje y comunicación y el decil de capital económico de los hogares de los estudiantes. Mientras que los alumnos que se ubican en el primer decil (10\% más pobres) ob- 
tienen en promedio una puntuación de 469 puntos, los estudiantes del décimo decil ( $10 \%$ más ricos) obtienen una puntuación de 537; en una escala de 200 a 800 unidades, con una media de 500 puntos. Esta diferencia de casi 70 puntos equivale a cerca de dos grados de escolaridad entre los dos grupos de estudiantes extremos.

FIGURA 5. Puntuaciones estandarizadas en Lenguaje y comunicación de estudiantes mexicanos según su condición socioeconómica: Educación Media Superior

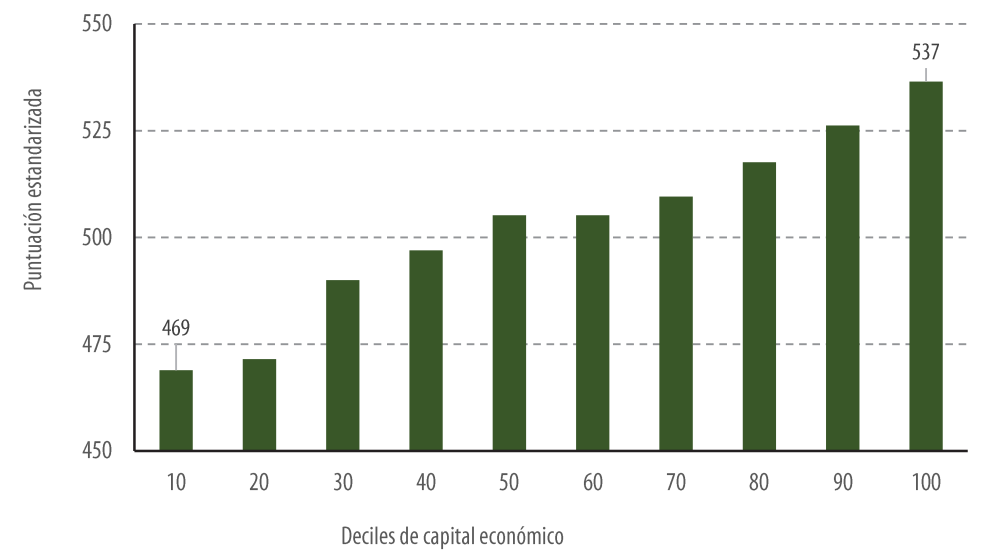

Fuente: reproducido de INEE. Informe 2018: La educación obligatoria en México. México: INEE.

\section{CONCLUSIONES}

Este artículo se propuso caracterizar en forma breve al Sistema Educativo Mexicano. Para ello, se analizó el contexto social en el que opera el SEN, el tamaño de las poblaciones escolares que atiende, la diversidad e inequidad de su oferta educativa, las características de sus docentes y los resultados de aprendizaje de sus estudiantes. Todas estas características, en conjunto, nos ofrecen una mirada de los grandes retos que enfrenta la educación obligatoria en México.

En principio, hay que reconocer que el SEN opera en un contexto social de pobreza e inequidad, donde la educación reprodu- 
ce las desigualdades sociales y económicas que se manifiestan en los diversos grupos poblacionales: el SEN ofrece una educación de menor calidad a los grupos más vulnerables. También es importante tomar en consideración el tamaño de la población que debe de atender el SEN, el crecimiento de las poblaciones según el grupo de edad idóneo para cursar los diversos niveles educativos y el tamaño de las poblaciones donde residen los escolares. Estas características dan una idea de los grandes retos del SEN para garantizar el derecho de todos los niños y jóvenes del país a recibir una educación de calidad con equidad.

Por otro lado, es importante también reconocer que el país ha avanzado considerablemente en algunos indicadores en materia de educación, tales como: el aumento en el nivel educativo de la población de 15 años a más, la reducción del analfabetismo y el incremento en la asistencia escolar en todos los niveles educativos, aunque en este último renglón, aún falta mucho por hacer en los niveles de preescolar y de EMS.

Uno de los aspectos en los que el país presenta mayores deficiencias es en la calidad de los aprendizajes de los estudiantes. Al término de la educación obligatoria, una tercera parte de ellos egresa sin dominar las habilidades de Lenguaje y comunicación que les permiten comprender cabalmente lo que leen. Asimismo, dos terceras partes de estos alumnos no dominan las habilidades matemáticas básicas, del Marco Curricular Común, que se centran en el manejo del álgebra y la solución de problemas. Además, la pobreza de los aprendizajes se relaciona estrechamente con las condiciones sociales y económicas de los estudiantes, de tal manera que es posible predecir el rendimiento académico de grupos de estudiantes según el decil del capital económico de sus hogares.

Finalmente, aunque en este texto no se abordó el tema de la reforma educativa, hay que reconocer que hubo avances considerables, tales como: un ordenamiento en los procesos de ingreso, promoción, permanencia y reconocimiento de los docentes a través de reglas claras, centradas en su mérito; el impulso de un nuevo modelo educativo más inclusivo, que pone énfasis en la escuela como eje central, renueva y articula los currículos de la educación obligatoria e incluye las competencias socioemocio- 
nales y las lenguas indígenas; $y$, la autonomía constitucional del INEE, que ha permitido diseñar la Política Nacional de Evaluación de la Educación y poner en marcha el Sistema Nacional de Evaluación Educativa, donde intervienen las autoridades federales y locales del país.

\section{REFERENCIAS BIBLIOGRÁFICAS}

Backhoff, E. y Pérez-Morán, J.C. (Coords.) (2015). Segundo Estudio Internacional sobre la Enseńanza y el Aprendizaje (TALIS, 2013): resultados de México, México: INEE.

Instituto Nacional para la Evaluación de la Educación (INEE) (2017). Panorama Educativo de México. México: INEE.

Instituto Nacional para la Evaluación de la Educación (INEE) (2018). Informe 2018: La educación obligatoria en México. México: INEE.

Instituto Nacional de Geografía y Estadística (INEGI)(2015). Principales resultados de la Encuesta Intercensal 2015. Estados Unidos Mexicanos. México: INEGI. 